\title{
OPEN Expression profiling of sexually dimorphic genes in the Japanese quail, Coturnix japonica
}

\author{
Miki Okuno ${ }^{1,5}$, Shuntaro Miyamoto ${ }^{2,5}$, Takehiko Itoh ${ }^{1}$, Masahide Seki ${ }^{3}$, Yutaka Suzuki $^{3}$, \\ Shusei Mizushima ${ }^{2,4}$ \& Asato Kuroiwa ${ }^{2,4}$
}

Research on avian sex determination has focused on the chicken. In this study, we established the utility of another widely used animal model, the Japanese quail (Coturnix japonica), for clarifying the molecular mechanisms underlying gonadal sex differentiation. In particular, we performed comprehensive gene expression profiling of embryonic gonads at three stages $(\mathrm{HH} 27, \mathrm{HH} 31$ and HH38) by mRNA-seq. We classified the expression patterns of 4,815 genes into nine clusters according to the extent of change between stages. Cluster 2 (characterized by an initial increase and steady levels thereafter), including 495 and $\mathbf{3 1 0}$ genes expressed in males and females, respectively, contained five key genes involved in gonadal sex differentiation. A GO analysis showed that genes in this cluster are related to developmental processes including reproductive structure development and developmental processes involved in reproduction were significant, suggesting that expression profiling is an effective approach to identify novel candidate genes. Based on RNA-seq data and in situ hybridization, the expression patterns and localization of most key genes for gonadal sex differentiation corresponded well to those of the chicken. Our results support the effectiveness of the Japanese quail as a model for studies gonadal sex differentiation in birds.

In birds, males are homogametic (ZZ) and females are heterogametic (ZW). The sex determination mechanism involving gene(s) on the sex chromosome is highly conserved among birds. In chickens, sex determination is thought to occur after embryonic day (E) 4.5, corresponding to Hamburger and Hamilton stage ${ }^{1} 24$ (HH24). After sex determination, the gonads differentiate to testes or ovaries according to the sex chromosomal constitution of cells. However, until around E6-6.5 (HH29), the gonads are considered "bipotential," indicating that they are able to differentiate into either testes or ovaries. At E6-6.5 (HH29), gonads begin morphological differentiation into testes in ZZ embryos or ovaries in ZW embryos. Sex-specific gonadal morphology emerges at this time, and genes important for ovary or testis development show sex-biased expression.

Genes involved in gonadal sex differentiation have been analysed extensively in chickens ${ }^{2,3}$. Several key genes involved in mammalian gonadal sex differentiation are conserved in chickens, including genes up-regulated in males (e.g., doublesex and mab-3-related transcription factor 1 (DMRT1), sex-determining region Y-box 9 (SOX9), anti-Mullerian hormone (AMH) and hemogen (HEMGN)), genes upregulated in females (e.g., forkhead box L2 (FOXL2) and cytochrome P450 family 19 subfamily A member 1 (CYP19A1, also known as aromatase)), and genes upregulated in both sexes (e.g., nuclear receptor subfamily 5 group A member 1 (NR5A1, also known as $S F-1))^{4-15}$. Gene expression has been evaluated in embryonic gonads from E4.5 (HH24) to E6 (HH29) by RNA-sequencing in the chicken ${ }^{16,17}$. These data indicated that over 1,000 genes are transcribed in a sex-biased manner at E6.5 (HH29), and the majority of these become biased between E4.5 (HH24) and E6 (HH29). Novel genes and pathways that are activated in a sex-specific manner at the time of gonadal sex differentiation have been identified, emphasizing the utility of the RNA-seq approach.

The chicken is a common animal model. A number of transgenic chickens have been generated by retroviral infection ${ }^{18-21}$ and transposon systems ${ }^{22,23}$. For several genes involved in gonadal sex differentiation, over-expression or knockdown experiments have been performed ${ }^{4,5,8-10,24,25}$. Genetic editing tools, such as transcription

\footnotetext{
${ }^{1}$ School of Life Science and Technology, Tokyo Institute of Technology, 2-12-1 Ookayama, Meguro-ku, Tokyo 152-8550, Japan. ${ }^{2}$ Biosystems Science Course, Graduate School of Life Science, Hokkaido University, Kita 10, Nishi 8, Kita-ku, Sapporo, Hokkaido 060-0810, Japan. ${ }^{3}$ Department of Computational Biology and Medical Sciences, The University of Tokyo, 5-1-5 Kashiwanoha, Kashiwa 277-8562, Japan. ${ }^{4}$ Division of Reproductive and Developmental Biology, Department of Biological Sciences, Faculty of Science, Hokkaido University, Kita 10 Nishi 8, Kita-ku, Sapporo, Hokkaido 060-0810, Japan. ${ }^{5}$ These authors contributed equally: Miki Okuno and Shuntaro Miyamoto. ${ }^{凶}$ email: asatok@sci.hokudai.ac.jp
} 
activator-like effector nuclease (TALEN) and clustered regulatory interspaced short palindromic repeats (CRISPR)/CRISPR-associated protein 9 (CRISPR/Cas9), have been applied to modify the chicken genome. Null mutants have been generated for two egg white genes, ovalbumin and ovomucoid ${ }^{26,27}$, as well as DEAD-box helicase 4 (DDX4; also known as vasa), which is essential for the proper formation and maintenance of germ cells $^{28}$. However, these methods are dependent on germline-mediated chimerism, which is time-consuming because individuals in the first generation are always mosaic. In addition, the introduction of mutations in female-specific genes on the $\mathrm{W}$ chromosome is nearly impossible because female-derived primordial germ cells are difficult to culture 29,30 .

We propose that the Japanese quail (Coturnix japonica) is a useful model for studies of the molecular mechanisms underlying gonadal sex differentiation. The Japanese quail is a small species in the family Phasianidae and is a well-established animal model for biological research. The Japanese quail benefits from its easier handling and shorter generation time than those of other Phasianidae species, such as the chicken. It requires only 16 days to hatch and 6 to 8 weeks of age to reach sexual maturity. In addition, the Japanese quail shows excellent reproductive performance (egg production, fertility and hatchability), comparable or superior to those of the chicken ${ }^{31}$. The Organization for Economic Co-operation and Development (OECD) recommends the use of the Japanese quail as a model for avian safety assessments ${ }^{32}$. Developing embryos of the Japanese quail can be easily cultured ex vivo and manipulated. In particular, it is worth noting that in vitro fertilization ${ }^{33,34}$ and complete culture from the single-cell stage to hatching ${ }^{35}$ have only been demonstrated in the Japanese quail to date. Current in vitro technologies in the species could be powerful tools for the production of genome-edited animals. Thus, the Japanese quail may be an effective alternative to the chicken as a laboratory research animal.

In this study, we performed comprehensive gene expression profiling of embryonic gonads at $\mathrm{HH} 27$ (just after sex determination and before morphological differentiation between ZZ and ZW embryonic gonads), HH31 (after morphological differentiation) and HH38 (proceeding differentiation) in the Japanese quail by mRNAseq. We further classified genes according to the extent of change between stages and performed GO analyses to establish the potential functions of these genes in reproduction. Our results demonstrate that expression profiling using RNA-seq data for embryonic gonads is effective for the identification of novel candidate genes involved in gonadal sex differentiation. Further analyses indicated that the expression levels and localization of key genes for gonadal sex differentiation were consistent with those in chicken embryos, except for HEMGN. Based on our findings, the Japanese quail is an effective model for the identification of novel genes involved in gonadal sex differentiation.

\section{Results}

Gene expression profiling. We conducted RNA-seq analysis focusing on changes in the expression level of genes. Total RNAs extracted form gonads of over than five individuals of each stage and sex were used. The RNA-seq data were used to obtain gene expression profiles for Japanese quail gonads at four developmental time points in males and females: HH27 (just after sex determination and before morphological differentiation between ZZ and ZW embryonic gonads), HH31 (after morphological differentiation), HH38 (proceeding differentiation) and adult. An MA-plot of TMM normalized FPKM is shown in Fig. S1. To confirm trends of gene expression patterns at each of the stages and chromosomes, the top 5,000 genes with high FPKM value at each samples are shown in Fig. 1, Fig. S2 and Table S1. We classified the top 5,000 genes into the following four groups according to fold change between sexes at the same stage: unbiased $(-\times 1.5$ fold change $), \times 1.5-2.0, \times 2.0-3.0$ and $\times 3.0$ - fold change. A proportion of genes with fold-change $\times 2.0$ - on the autosomes was confirmed to be higher in the adult testis (43.1\%) and ovary (36.6\%) than at the other three early developmental time points. We also confirmed that the tendency did not change even if the threshold value of fold-change $(\times 1.5$ or $\times 3.0)$ was changed. In males, genes located on the $\mathrm{Z}$ chromosome showed a similar pattern to that of autosomal genes, and the proportion of $Z$ chromosomal genes with fold-change $\times 2.0$ - was higher than that on the autosomes in all developmental stages. It was also confirmed that the proportion of genes with fold change $\times 2.0-(\times 1.5-$ and $\times 3.0$, too) on chromosome $\mathrm{Z}$ is higher in males than in females at all stages. In particular, genes with fold-change $\times 2.0$ accounted for $63.8 \%$ and $23.6 \%$ of $\mathrm{Z}$ chromosomal genes in adult males and females, respectively, and likewise genes with fold-change $\times 1.5$ - accounted for $79.4 \%$ and $34.0 \%$, respectively. The top 3,000 and 10,000 genes showed similar features as the top 5,000 genes (Table S2 and S3). We also confirmed that similar results were obtained using GFOLD and isoDE2, which are designed for gene expression analysis using RNA-seq data without biological replicates (Figs. S3 and S4).

Focusing on the 4,815 genes with TMM normalized FPKM values of $\geq 30$, we classified expression patterns into nine clusters according to the extent of change in two comparisons, HH27-HH31 and HH31-HH38 (Table 1 and Fig. 2; $3 \times 3$ clusters). Cluster 2 (up-flat), including 495 and 310 genes expressed in males and females, respectively, contained several genes involved in gonadal sex differentiation, including DMRT1, SOX9 and $A M H$ in males (blue lines in cluster 2, Fig. 2), and FOXL2 and CYP19A1 in females (orange lines in cluster 2, Fig. 2). These genes displayed a common trend in which the expression level increased during HH27-HH31 and remained constant thereafter. Among the genes classified into cluster 2, those whose fold-change between males and females was $\geq 2$ at HH31 comprised 52 genes in males (Table S4) and 40 genes in females (Table S5). NR5A1, which activates CYP19A1 in the chicken ovary, was assigned to cluster 5 (flat-flat) for females. HEMGN, involved in testis differentiation in the chicken, was excluded from the clustering analysis owing to its low expression level in the Japanese quail.

GO analysis. To investigate the functional characteristics of the 92 genes with expression differences between males and females in cluster 2, we performed a GO analysis. In total, 13,243 of the 16,037 protein-coding genes of the Japanese quail were annotated to one or more GO terms. The criteria for significance were FDR $<0.05$ and 
Male expressed genes (autosome)

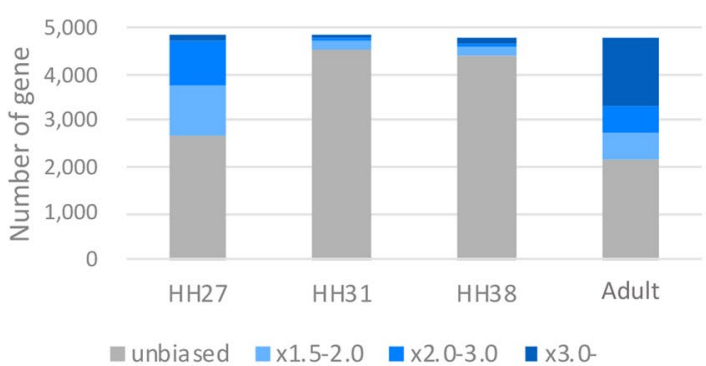

Female expressed genes (autosome)

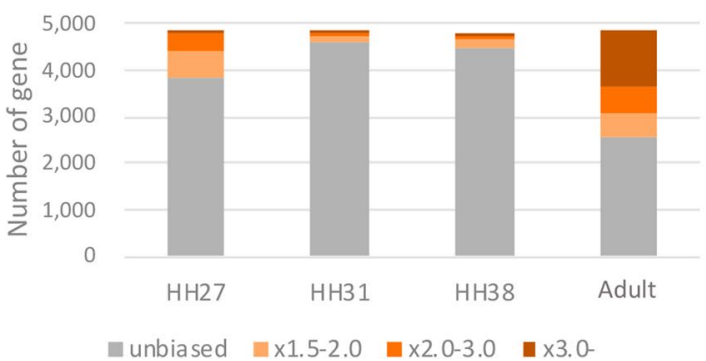

Male expressed genes (chr Z)

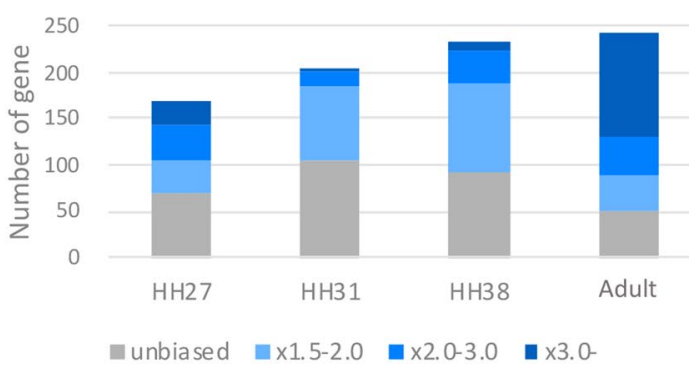

Female expressed genes (chr Z)

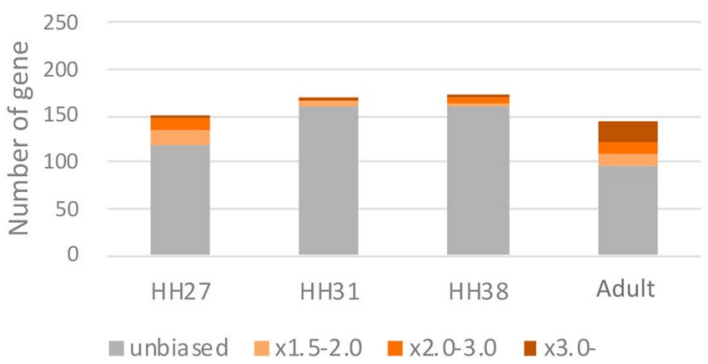

Figure 1. Top 5,000 genes with high FPKM value in male (ZZ) and female (ZW) at the following four stages: embryonic gonads at $\mathrm{HH} 27, \mathrm{HH} 31$, and $\mathrm{HH} 38$, and the adult testis or ovary. Bar graphs were showed separately for autosomes (left side) or the $\mathrm{Z}$ chromosome (right side). Genes were classified into the following four groups according to fold change between sexes at the same stage: unbiased (grey), $\times 1.5-2.0$ fold change (light blue for male and light orange for female, respectively), $\times 2.0-3.0$ (blue and orange), and $\times 3.0$ - (dark blue and brown).

\begin{tabular}{|l|l|l|l|l|l|l|l|}
\hline \multirow{2}{*}{ Cluster } & \multicolumn{3}{l|}{ Expression pattern } & \multicolumn{2}{l|}{ Number of genes } & \multicolumn{2}{l|}{ Genes known to be involved in sex differentiation } \\
\cline { 2 - 9 } & HH27-31 & HH31-38 & Male & Female & Total & Male & Female \\
\hline Cluster 1 & $\nearrow$ & $\nearrow$ & 320 & 192 & 512 & & \\
\hline Cluster 2 & $\nearrow$ & $\rightarrow$ & 495 & 310 & 805 & DMRT1, SOX9, AMH & FOXL2, CYP19A1, AMH \\
\hline Cluster 3 & $\nearrow$ & $\searrow$ & 558 & 145 & 703 & & DMRT1 \\
\hline Cluster 4 & $\rightarrow$ & $\nearrow$ & 363 & 974 & 1,337 & & \\
\hline Cluster 5 & $\rightarrow$ & $\rightarrow$ & 139 & 1,353 & 1,492 & FOXL2, CYP19A1 & NR5A1 \\
\hline Cluster 6 & $\rightarrow$ & $\searrow$ & 562 & 1,282 & 1,844 & & SOX9 \\
\hline Cluster 7 & $\searrow$ & $\nearrow$ & 507 & 178 & 685 & & \\
\hline Cluster 8 & $\searrow$ & $\rightarrow$ & 1,090 & 256 & 1,346 & & \\
\hline Cluster 9 & $\searrow$ & $\searrow$ & 781 & 125 & 906 & NR5A1 & \\
\hline & & Total & 4,815 & 4,815 & 9,630 & & \\
\hline
\end{tabular}

Table 1. Gene expression pattern in embryonic gonads of Japanese quail.

a hierarchical depth of up to three. Focusing on the biological process domain, 92 genes (52 in males and 40 in females) were assessed. GO terms related to developmental processes including reproductive structure development and developmental processes involved in reproduction were significant (Table 2).

Genes with dimorphic expression involved in gonadal sex differentiation. We analysed the expression patterns of seven genes involved in gonadal sex differentiation, DMRT1, SOX9, AMH, HEMGN, NR5A1, FOXL2 and CYP19A1, using RNA-seq data. We obtained RNA-seq data from the embryonic gonads of the Japanese quail at $\mathrm{HH} 27, \mathrm{HH} 31$ and $\mathrm{HH} 38$ and from the testis and ovary of an adult for comparisons between males (ZZ) and females (ZW). FPKM values normalized using the TMM method for seven genes were evaluated (Fig. 3).

DMRT1 expression was detected at $\mathrm{HH} 27$ and reached a peak at HH31 in males and females (Fig. 3A). Throughout all examined stages, expression levels were higher in males than in females. DMRT1 expression remained high in adult males, unlike the other six genes, which decreased over time. SOX 9 expression was slightly higher in females than in males at HH27 (Fig. 3B). Beyond HH27, expression increased in males but remained at extremely low levels in females. Obvious expression of $A M H$ was not detected at $\mathrm{HH} 27$; however, 

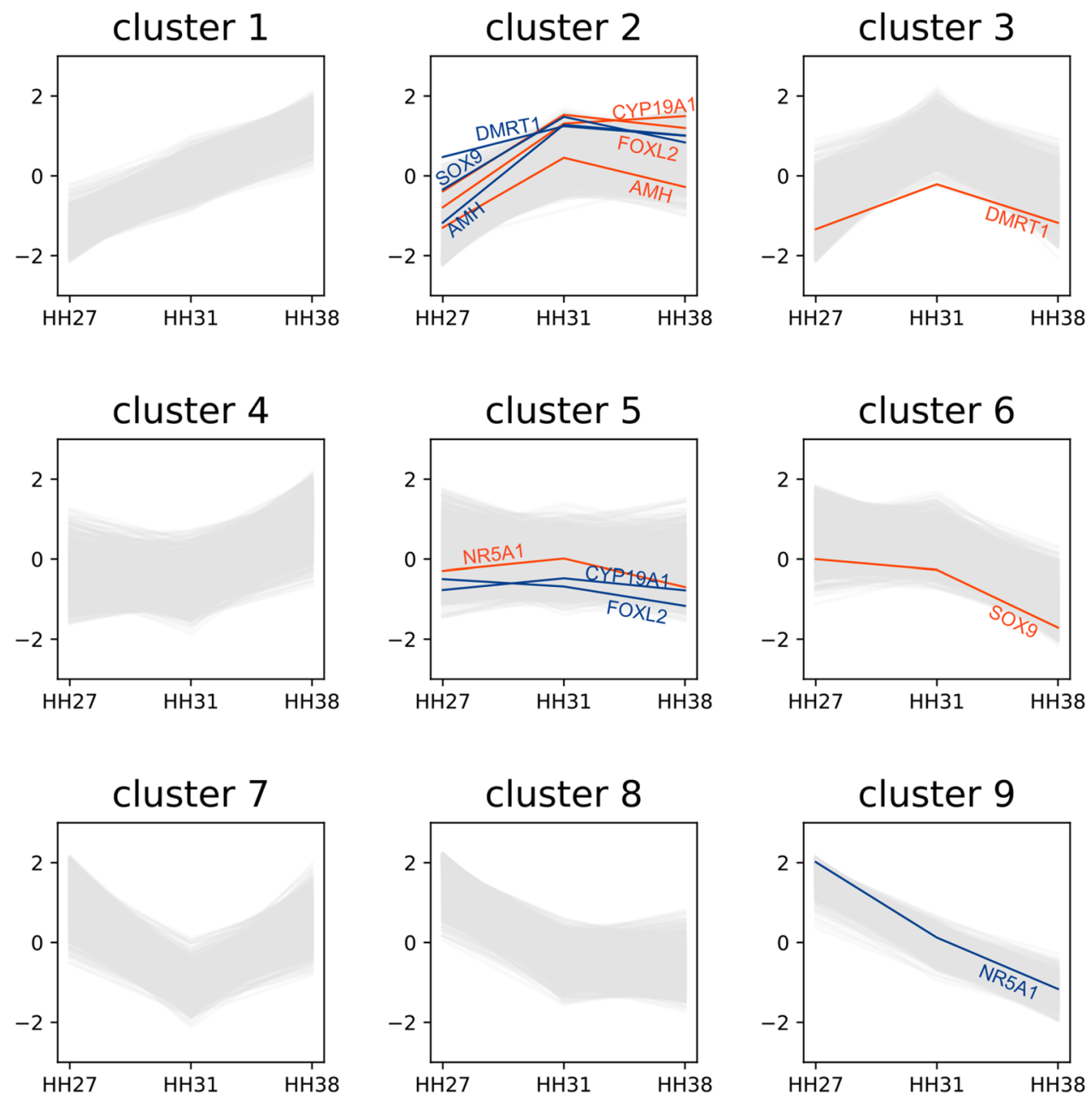

Figure 2. Expression patterns of nine clusters during gonadal sex differentiation. Between $\mathrm{HH} 27$ and $\mathrm{HH} 31$, gene expression levels increased in clusters 1, 2 and 3, remained constant in clusters 4, 5 and 6, and decreased in clusters 7, 8 and 9. Similarly, between $\mathrm{HH} 31$ and $\mathrm{HH} 38$, gene expression levels increased in clusters 1, 4 and 7 , remained the same in clusters 2,5 and 8 , and decreased in clusters 3,6 and 9 . Blue and orange lines indicate genes related to gonadal differentiation in males (ZZ) and females (ZW), respectively.

\begin{tabular}{|l|l|l|l|l|l|}
\hline \# GO & Term & Ratio_in_study & Ratio_in_pop & Depth & p_fdr \\
\hline GO:0,048,608 & reproductive structure development & $7 / 92$ & $99 / 20,132$ & 3 & 0.000 \\
\hline GO:0,048,856 & anatomical structure development & $21 / 92$ & $1279 / 20,132$ & 2 & 0.002 \\
\hline GO:0,032,502 & developmental process & $26 / 92$ & $2064 / 20,132$ & 1 & 0.002 \\
\hline GO:0,003,006 & developmental process involved in reproduction & $8 / 92$ & $207 / 20,132$ & 2 & 0.008 \\
\hline GO:0,048,869 & cellular developmental process & $18 / 92$ & $1229 / 20,132$ & 2 & 0.014 \\
\hline GO:0,050,793 & regulation of developmental process & $17 / 92$ & $1116 / 20,132$ & 3 & 0.014 \\
\hline GO:0,051,239 & regulation of multicellular organismal process & $18 / 92$ & $1291 / 20,132$ & 3 & 0.020 \\
\hline
\end{tabular}

Table 2. GO terms in the "cluster 2 " genes exhibiting different expression levels between males and females.

expression increased dramatically in males after HH31 (Fig. 3C). In adults, although the AMH expression level was low, it was higher in females than in males. Although HEMGN expression was slightly higher in males than in females at $\mathrm{HH} 38$, it was quite low at all embryonic stages in both sexes (Fig. 3D). In adults, HEMGN expression was only detected in females.

NR5A1 expression was approximately two-fold higher in males than in females at HH27 (Fig. 3E). NR5A1 expression was similar in males and females at $\mathrm{HH} 31$, since expression in males decreased. In adults, the expression pattern was reversed, with higher levels in females than in males. FOXL2 and CYP19A1 showed femalespecific expression throughout all examined stages (Fig. $3 \mathrm{~F}$ and $\mathrm{G}$ ). A peak of FOXL2 expression was detected at embryonic stage $\mathrm{HH} 31$, and the highest expression among all stages was observed in adult females. CYP19A1 
(A) DMRT1

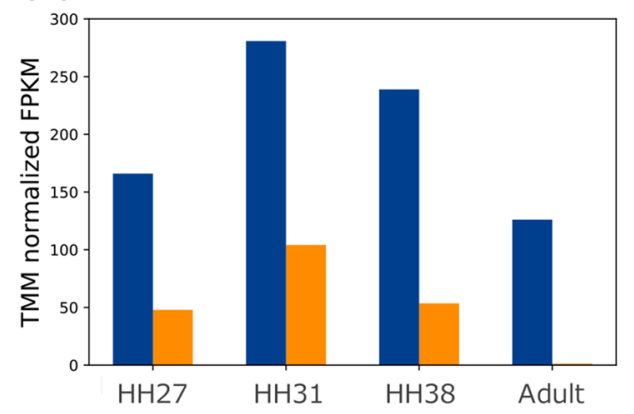

(B) $50 \times 9$

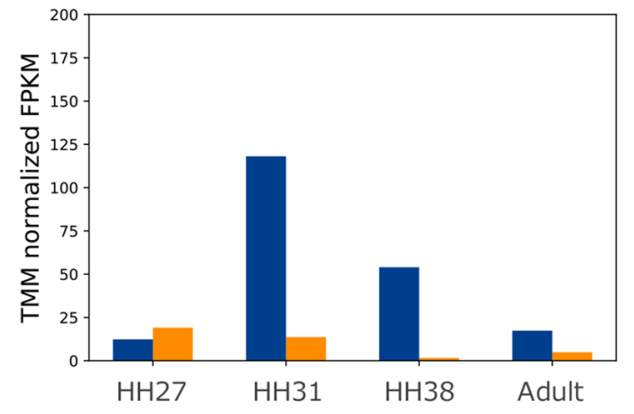

(C) $A M H$

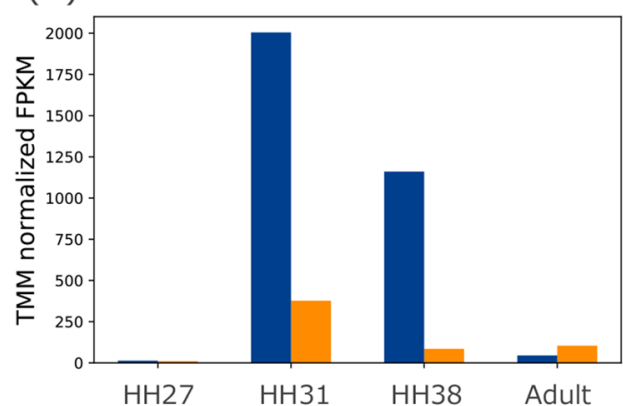

(D) HEMGN

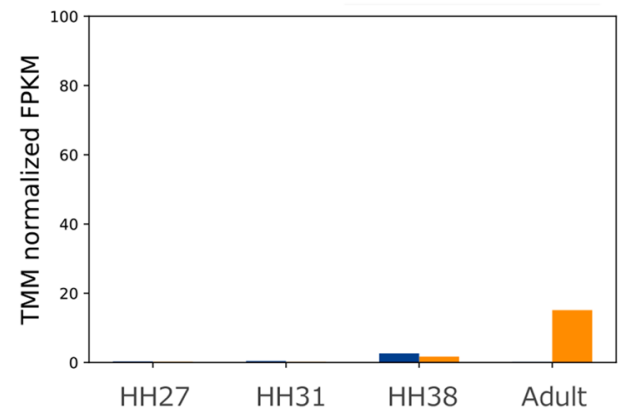

(E) $N R 5 A 1$

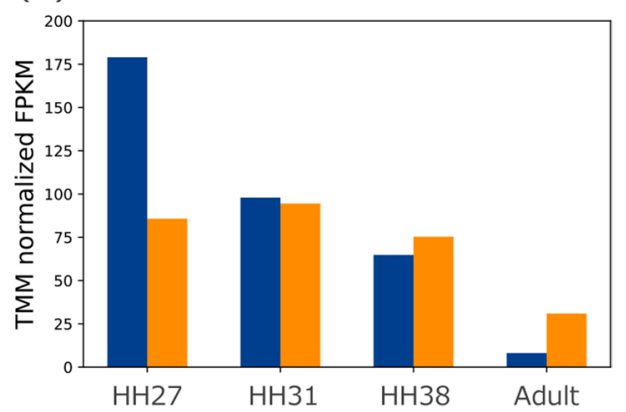

(F) FOXL2

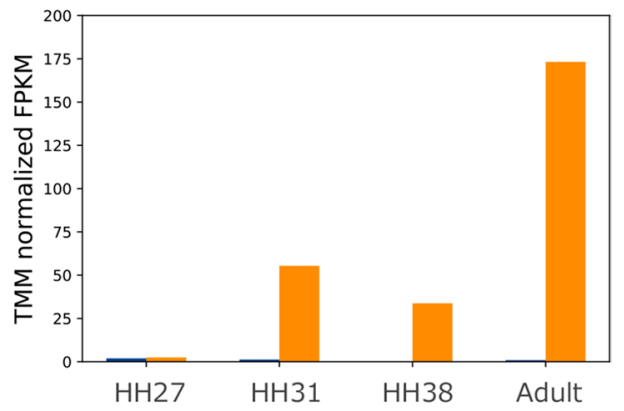

(G) CYP19A1

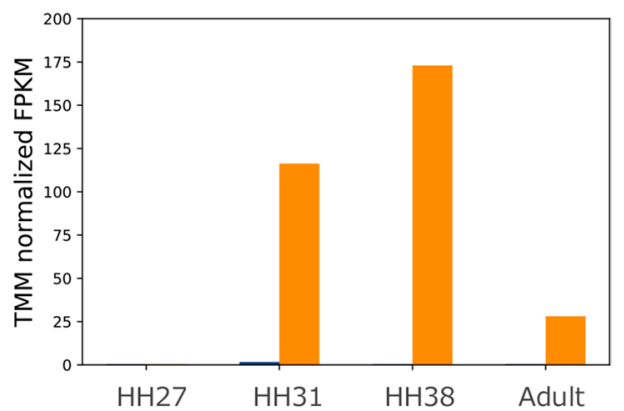

Figure 3. Comparison of expression patterns of seven genes between male $(\mathrm{ZZ})$ and female $(\mathrm{ZW})$ gonads using TMM normalized FPKM values. The expression patterns of DMRT1 (A), SOX9 (B), AMH (C), HEMGN (D), NR5A1 (E), FOXL2 (F) and CYP19A1 (G) are shown based on TMM normalized FPKM values in embryonic gonads at $\mathrm{HH} 27, \mathrm{HH} 31, \mathrm{HH} 38$ and the adult testis or ovary. Blue and orange bars indicate males (ZZ) and females (ZW), respectively. 
expression was higher than FOXL2 expression during embryonic stages, with a peak at HH38. Adult females showed low expression of CYP19A1.

We used in situ hybridization to confirm the localization of these seven genes in Japanese quail embryonic gonads at five developmental stages, $\mathrm{HH} 27, \mathrm{HH} 29, \mathrm{HH} 31, \mathrm{HH} 34$ and $\mathrm{HH} 38$. DMRT1 signals were localized to the gonadal medulla of males (ZZ) at all examined stages (Fig. 4A) and in the gonadal medulla of females (ZW) from $\mathrm{HH} 27$. However, the signals were weaker in females than in males and almost undetectable after HH34. SOX9 signals were detected in the gonadal medulla of males from HH31 (Fig. 4B) and were not detected in female gonads at any stages. A similar pattern was observed for $A M H$ hybridization in male gonads (Fig. 4C); however, the signals in males were the strongest among the three male-biased genes. Although the signals were weak in female gonads at all stages, expression was detected at HH31, 34 and 38 (arrowheads in Fig. 4C). In HEMGN, quite weak signals were observed in males from HH29 to HH38 (Fig. 4D). DMRT1 showed the earliest expression (from $\mathrm{HH} 27$ ) among all four male-biased genes.

Relatively strong NR5A1 signals were observed in the gonadal medulla of males and females at all examined stages (Fig. 4E). Two female-biased genes, FOXL2 and CYP19A1, showed female-specific expression (Fig. 4F and $\mathrm{G}$, respectively). FOXL2 was detected earlier than CYP19A1 (i.e., HH29 and HH31, respectively). The signals of both genes were localized to the gonadal medulla of females, but CYP19A1 expression was stronger than FOXL2 expression. Overall, the mRNA expression and localization patterns were consistent with the patterns reported in chicken embryos, with the exception of HEMGN.

\section{Discussion}

Genes involved in gonadal sex differentiation show sex-biased expression and regulate testicular or ovarian development. Several of these genes have been evaluated in knockout mouse models and in humans with disorders of sex development. However, gonadal sex differentiation is not well-understood and likely involves a number of unknown genes. In birds, the molecular mechanism underlying gonadal sex differentiation has implications for poultry breeding. We found that embryonic gonads at $\mathrm{HH} 27$ (just after sex determination) show the highest percentage of genes with sex-biased expression on the autosomes in the three embryonic stages. Many unknown genes are differentially expressed to promote gonadal differentiation after sex determination. The number of $Z$ chromosomal genes showed male-biased expression was higher than that on the autosomes in all stages. We also confirmed that male-biased expression genes distributed throughout the $\mathrm{Z}$ chromosome, and was not biased to small regions (Fig. S5). This indicates that $\mathrm{Z}$ chromosomal genes are not completely dosage compensated in birds. Previous studies have reported that the mean expression levels of Z-linked genes are 1.6-fold higher in males than in females ${ }^{36-38}$. A similar result has been obtained in an RNA-seq analysis of blastoderms and embryonic gonads of chickens ${ }^{15}$. We could not identify $\mathrm{W}$ genes owing to insufficient sequence information for the Japanese quail. The enrichment of genome sequence information for the $\mathrm{W}$ chromosome of the species should be a focus of research.

We classified expression patterns into nine clusters according to the extent of change between HH27 and $\mathrm{HH} 31$ and between HH31 and HH38. Five genes involved in gonadal sex differentiation, DMRT1, SOX9 and $A M H$ in males and FOXL2 and CYP19A1 in females, were assigned to cluster 2 (up-flat). These results indicate that genes involved in gonadal sex differentiation tend to increase after sex determination (HH27-HH31) and remain at a constant level during subsequent sex differentiation (HH31-HH38). Genes in cluster 2 were associated with GO terms related to developmental processes, including reproductive structure development, anatomical structure development, developmental process, developmental processes involved in reproduction, cellular developmental process and regulation of developmental process. Our expression profiling approach using RNA-seq data for embryonic gonads was effective for the identification of candidate genes not known to function on gonadal sex differentiation. In particular, the genes classified into cluster 2 ( 52 and 40 genes in males and females, respectively) (Table S4 and S5) are candidates for further functional assays.

The best candidate master testis-determining gene is Z-linked DMRT1 in birds lacking mammalian sex determinant Sry/SRY (sex-determining region Y). The chicken homolog of DMRT1 is expressed more highly in undifferentiated gonads of males than in females, and a high expression level is maintained in male gonads during testis development. In the Japanese quail, we also detected higher DMRT1 expression in male embryonic gonads at $\mathrm{HH} 27$, which was the earliest expressed male-biased genes, and the expression continued after HH27. These results indicate that $D M R T 1$ is a conserved gene with key functions in testis determination in the Japanese quail. We also observed male-biased expression of $S O X 9$ and $A M H$ in embryonic gonads after $\mathrm{HH} 31$ in the Japanese quail. In placental mammals, SRY directly activates Sox9/SOX9 by binding to the Sox9/SOX 9 enhancer together with NR5A1 in the undifferentiated gonads of XY embryos ${ }^{39}$. Subsequently, SOX9 directly regulates $A m h / A M H$ expression by binding to the $A m h / A M H$ proximal promoter together with other transcription factors ${ }^{40-47}$. However, in chickens, there is a time-lag between the initial expression of DMRT1 and SOX9, which occur at days 3.5 and 6.5, respectively ${ }^{48}$. Furthermore, $A M H$ mRNA is expressed prior to $S O X 9 \mathrm{mRNA}^{7}$. Therefore, other factors, probably chicken (birds)-specific factors, must be components of the molecular cascade between DMRT1 and SOX9. We further found that SOX9 is expressed later than DMRT1 and AMH in the Japanese quail, indicating that the molecular cascade between these genes is conserved in the two species.

By contrast, we did not detect significant HEMGN expression in Japanese quail gonads. HEMGN is located on the $\mathrm{Z}$ chromosome and was first reported as a chicken-specific factor for testis differentiation by mediating SOX9 regulation under $D M R T 1^{10}$. In mice, Hemgn (also known as EDAG in humans) is a hematopoietic tissue-specific gene encoding a nuclear protein ${ }^{49}$. Although the gene is not expressed in the gonads during embryogenesis in mammals, HEMGN is expressed in hematopoietic tissues and in early embryonic gonads of male chickens ${ }^{10}$. In ZW embryonic gonads masculinized by aromatase inhibitor treatment, HEMGN expression is induced. ZW embryos overexpressing HEMGN have masculinized gonads with increases in the male marker genes DMRT1 


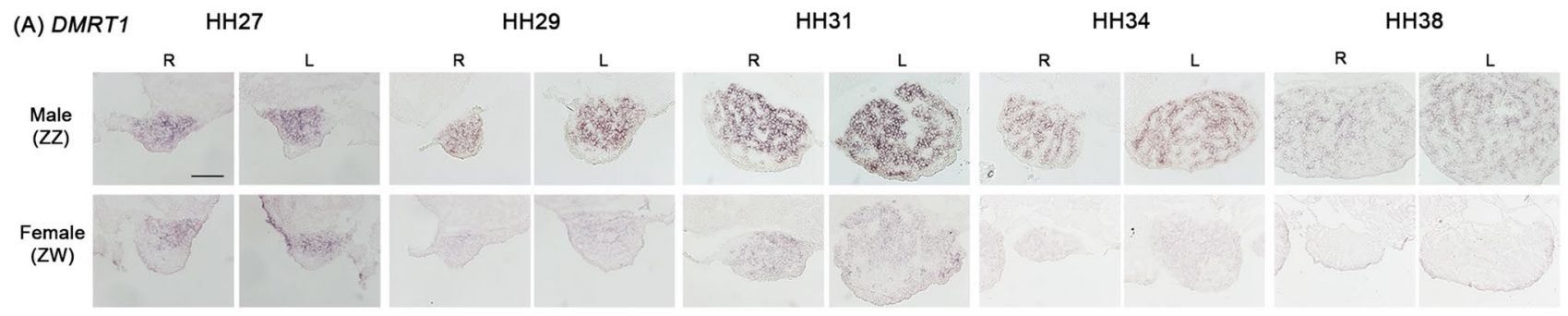

(B) $50 \times 9$

\section{Male}

(ZZ)

Female

(ZW)

(C) $A M H$
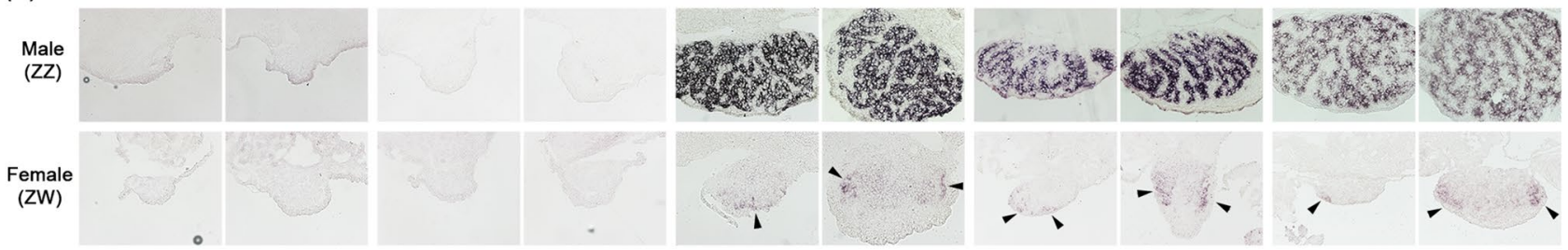

(D) HEMGN
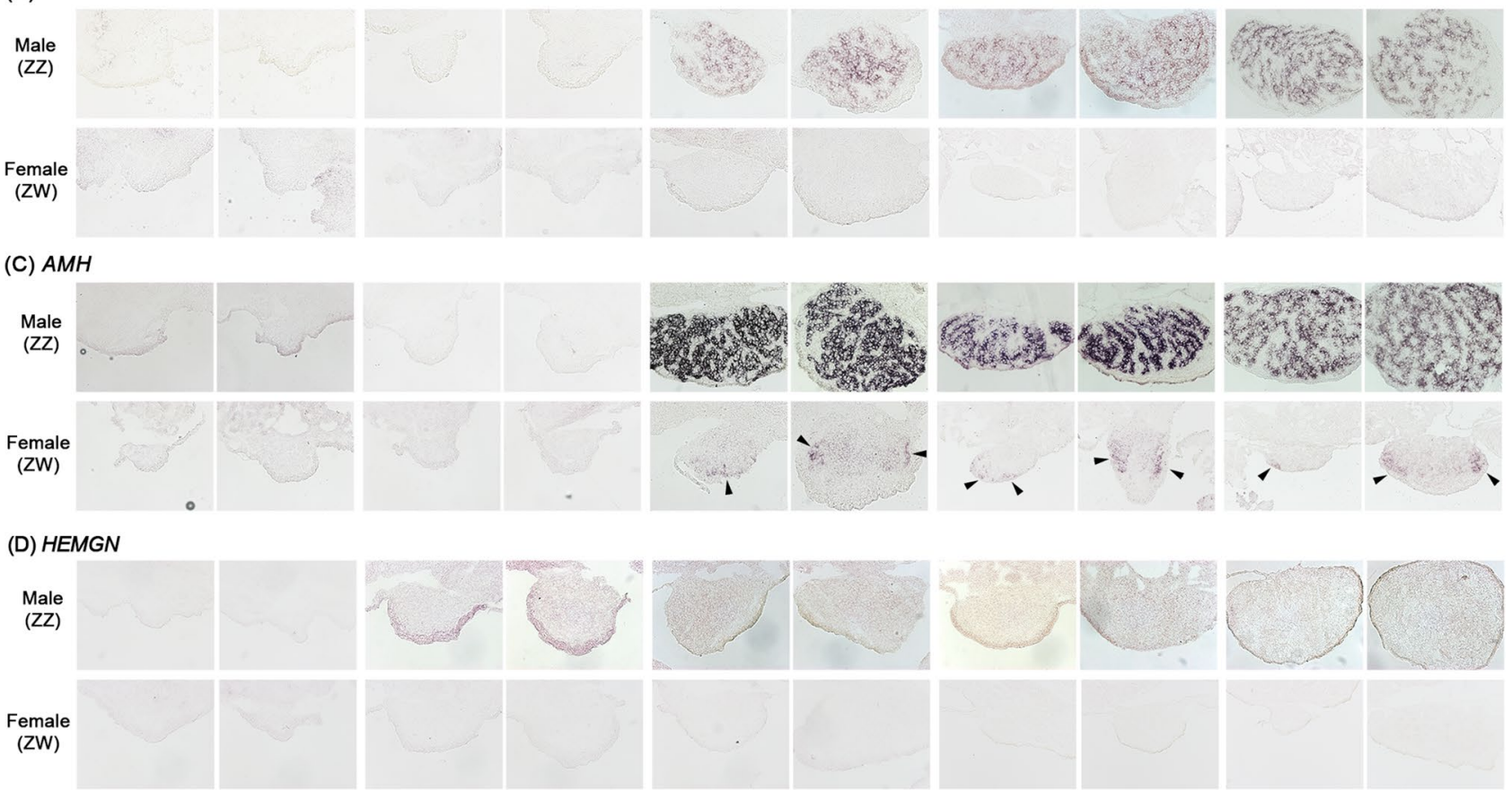

(E) NR5A1
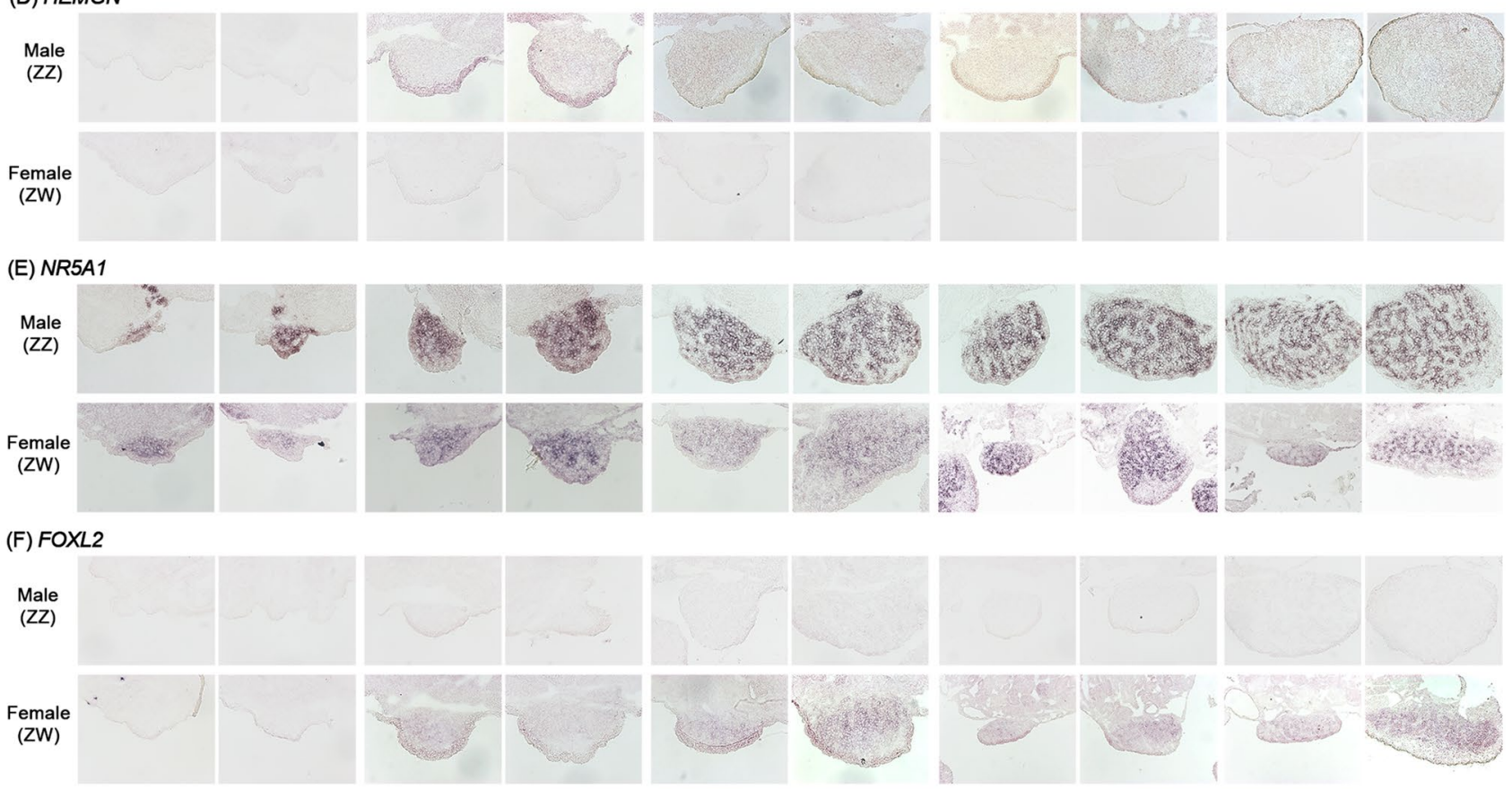

(G) CYP19A1
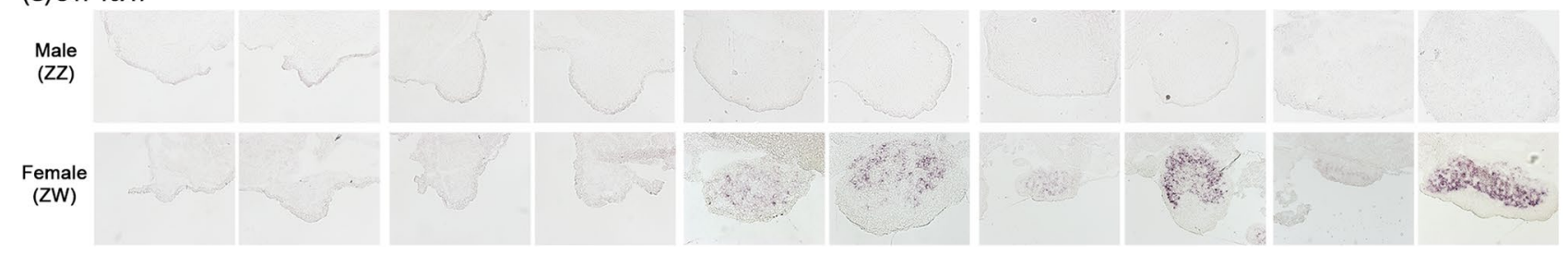

Figure 4. Expression patterns of seven genes in embryonic gonads of the Japanese quail. Expression patterns of DMRT1 (A), SOX9 (B), AMH (C), HEMGN (D), NR5A1 (E), FOXL2 (F) and CYP19A1 (G) were examined by in situ hybridization in frozen gonad sections of ZZ (male) and ZW (female) at HH27, HH29, HH31, HH34 and $\mathrm{HH} 38$. R and L indicate right and left gonads, respectively. Scale bar indicates $100 \mu \mathrm{m}$. 
and SOX9 and decreases in the female marker genes aromatase and FOXL2. Furthermore, the distribution of germ cells shows a testis-like pattern. These findings suggest that HEMGN is specifically involved in gonadal differentiation in the chicken. However, HEMGN mRNA expression has not been detected in embryonic gonads of the emu and zebra finch ${ }^{50}$. We confirmed a lack of detectable HEMGN expression in the Japanese quail, belonging to the order Galliformes, along with the chicken, although the identity of mRNA sequences of HEMGN is 86.24\% between chicken (XM_430508) and Japanese quail (XM_032441476). Accordingly, the role of this gene in gonadal sex differentiation could be limited to the chicken and closely related species.

FOXL2 is a conserved gene involved in ovary development in vertebrates ${ }^{13-15}$. The expression patterns of FOXL2 and CYP19A1 are highly correlated in the developing ovary in chickens ${ }^{12}$. CYP19A1 is also important for ovary development because it encodes an enzyme (aromatase) responsible for converting androgens to estradiol. FOXL2 is expressed just prior to CYP19A1, suggesting that it directly or indirectly regulates aromatase transcription in chickens. We observed nearly identical expression patterns in the Japanese quail. FOXL2 was initially detected at $\mathrm{HH} 29$, before the expression of CYP19A1. These results indicate that the functions of two female-biased genes in ovary development are conserved in the Japanese quail. All examined genes other than HEMGN showed similar expression and localization patterns in embryonic gonads to those in the chicken, suggesting functional conservation. Our results confirm that these genes can be used as molecular markers for studies of gonadal sex differentiation in the Japanese quail. More broadly, our gene profiling and expression analyses are expected to contribute to the identification of novel candidate genes involved in gonadal sex differentiation in birds.

\section{Materials and methods}

Animals and ethics statement. Fertilized Japanese quail eggs were purchased from Motoki Corporation (Saitama, Japan). Fertilized eggs were incubated at $37.5^{\circ} \mathrm{C}$. The sex of each embryo was determined by PCR genotyping using genomic DNA as the template ${ }^{51}$. The developmental stage of each embryo was determined based on a previous report by Ainsworth et al..$^{52}$.

All animal experiments described in this study were approved by the Institutional Animal Care and Use Committee of National University Corporation Hokkaido University and were performed in accordance with the Guidelines for the Care and Use of Laboratory Animals issued by Hokkaido University. This study did not involve any human participants or specimens.

RNA extraction and cDNA synthesis. Total RNA was extracted from embryonic gonads of the Japanese quail at $\mathrm{HH} 27, \mathrm{HH} 31, \mathrm{HH} 38$, and adult testis and ovary using an RNeasy Kit (Qiagen, Hilden, Germany) according to the manufacturer's instructions. We used left gonads of embryo and left testes and ovaries of adult, because Japanese quail shows asymmetric development of gonads that right gonads are gradually depress and fail to develop in ZW female. RNA was treated with DNase I and reverse-transcribed using SuperScript III reverse transcriptase (Thermo Fisher Scientific, Waltham, MA, USA) and an oligo(dT) primer.

RNA-seq. Total RNA was quantified using a Bioanalyzer and RNA 6000 Nano Kit (Agilent, Santa Clara, CA, USA). Total RNA with an RIN value exceeding 9.7 was used for subsequent experiments. Using $200 \mathrm{ng}$ of total RNA as an input, the libraries for RNA-seq were prepared using the SureSelect Strand-Specific RNA Library Preparation Kit (Agilent) according to the manufacturer's protocol. Then, 100-bp paired-end reads were obtained using the HiSeq3000 platform (Illumina, San Diego, CA, USA). Sequencing data have been deposited in the DDBJ Sequence Read Archive under accession numbers DRA007208 and DRA008738.

Gene expression profiling. Total RNAs were extracted from gonads of over than five individuals of each stage and sex. We conducted RNA-seq analysis using the extracted RNAs focusing on changes in the expression level of genes. Firstly, for the following analyses, low-quality and adaptor sequences in RNA-seq reads were removed using Platanus_trim v1.0.7 (https://platanus.bio.titech.ac.jp/pltanus_trim). Fragments per kilobase of transcript per million mapped reads (FPKM) were estimated using RSEM $(1.3 .0)^{53}$. We used RefSeq Japanese quail genome (Coturnix japonica 2.1, GCF_001577835.1) eliminating a mitochondrial sequence as a reference genome. In RSEM, RNA-seq reads were mapped to the reference genome using bowtie2-2.3.4 (-bowtie2-mismatch-rate 0.03$)^{54}$. Next, to enable make comparisons of FPKM values between samples, we applied the trimmed mean of M-values (TMM) normalization method for FPKM values. The TMM normalized FPKM values were calculated using TCC (3.9) ${ }^{55}$. To confirm gene expression patterns at each of the stages or at autosome and chromosome Z, we classified top 5,000 genes with high FPKM value into the following four groups according to fold change between sexes at the same stage: unbiased $(-\times 1.5$ fold change $)$ and $\times 1.5-2.0, \times 2.0-3.0$ and $\times 3.0-$ fold change. To confirm statistically supported gene expression and fold changes between sexes, we also ran two existing tools, GFOLD v1.1.4 $4^{56}$ and isoDE2 v1.1.5 $5^{57}$ with default parameters. The two tools were designed for gene expression analysis using RNA-seq data without biological replicates.

Genes were classified according to their expression patterns at three stages (HH27, HH31 and HH38). The target genes for classification had $\geq 30$ TMM normalized FPKM in at least one of six samples (males and females at three stages). The TMM normalized FPKM values were pre-processed according to the common practice of applying quantile normalization, taking the logarithm of data values and converting $\mathrm{z}$-scores. To confirm differences of expression pattern between males and females, we normalized FPKM values of males and at once females for each gene. Based on the difference in the normalized values between $\mathrm{HH} 27$ and $\mathrm{HH} 31$, the genes in each sex were divided into three by k-means clustering using Euclidean distance (conducted by sklearn library v0.19.2, Python v3.7.0). By this clustering, the differences between the two stages were classified into the following three patterns: increase (up), no change (flat) and decrease (down). Similarly, classification was also performed 
between $\mathrm{HH} 27$ and $\mathrm{HH} 38$, and the genes were finally classified into nine groups according to the combination of the differences between $\mathrm{HH} 27$ and $\mathrm{HH} 31$ and between $\mathrm{HH} 31$ and $\mathrm{HH} 38$.

Gene ontology (GO) analysis. To obtain GO terms associated with Japanese quail genes, GO terms for homologous RefSeq chicken genes (GCF_000002315.4) were obtained. GO terms predicted using InterProScan $(5.33-72.0)^{58}$ for Japanese quail protein sequences were merged with the previous GO terms obtained for chicken genes. Next, a GO enrichment analysis was conducted using goatools $(0.9 .5)^{59}$. Enriched GO terms with a false discovery rate (FDR) of $<0.05$ and hierarchical depth of up to three in a sub-ontology, biological process (BP), were obtained.

In situ hybridization. A fragment of each gene was amplified by RT-PCR using cDNA obtained from gonads as the template. The sequences of primers are listed in Table S6. RNA extraction and cDNA synthesis were performed as described earlier. The CDS sequence of chicken NR5A1 was inserted in a pBluescript SK. The PCR products of other genes were subcloned using the pGEM T-Easy Vector System (Promega, Madison, WI, USA). cDNA clones were labelled using Digoxigenin RNA Labeling Mix (Roche, Basel, Switzerland) and T7, SP6 or T3 RNA polymerase (MAXIscript; Thermo Fisher Scientific). Hybridization to serial frozen sections was performed as described previously ${ }^{23}$. The incubation temperature was modified to $65-70{ }^{\circ} \mathrm{C}$. Images were captured using a cooled CCD camera (DS-Ri1; Nikon, Tokyo, Japan) mounted on a Nikon ECLIPSE E800 microscope and were analysed using NIS ELEMENTS (Nikon).

Received: 13 February 2020; Accepted: 6 November 2020

Published online: 30 November 2020

\section{References}

1. Hamburger, V. \& Hamilton, H. L. A series of normal stages in the development of the chick embryo. Dev. Dyn. 195, 231-272 (1992).

2. Kuroiwa, A. Sex-determining mechanism in avians. Adv. Exp. Med. Biol. 1001, 19-31 (2017).

3. Hirst, C. E., Major, A. T. \& Smith, C. A. Sex determination and gonadal sex differentiation in the chicken model. Int. J. Dev. Biol. 62, 153-166 (2018).

4. Smith, C. A. et al. The avian Z-linked gene DMRT1 is required for male sex determination in the chicken. Nature 461, 267-271 (2009).

5. Lambeth, L. S. et al. Over-expression of DMRT1 induces the male pathway in embryonic chicken gonads. Dev. Biol. 389, 160-172 (2014).

6. Morais da Silva, S. et al. Sox9 expression during gonadal development implies a conserved role for the gene in testis differentiation in mammals and birds. Nat. Genet. 14, 62-8 (1996).

7. Oreal, E. et al. Early expression of AMH in chicken embryonic gonads precedes testicular SOX9 expression. Dev. Dyn. 212, 522-532 (1998).

8. Lambeth, L. S. et al. Anti-müllerian hormone is required for chicken embryonic urogenital system growth but not sexual differentiation. Biol. Reprod. 93, 138 (2015).

9. Lambeth, L. S. et al. Overexpression of anti-Müllerian hormone disrupts gonadal sex differentiation, blocks sex hormone synthesis, and supports cell autonomous sex development in the chicken. Endocrinology 157, 1258-1275 (2016).

10. Elbrecht, A. \& Smith, R. G. Aromatase enzyme activity and sex determination in chickens. Science 255, 467-470 (1992).

11. Govoroun, M. S. et al. Isolation of chicken homolog of the FOXL2 gene and comparison of its expression patterns with those of aromatase during ovarian development. Dev. Dyn. 231, 859-870 (2004).

12. Loffler, K. A., Zarkower, D. \& Koopman, P. Etiology of ovarian failure in blepharophimosis ptosis epicanthus inversus syndrome: FOXL2 is a conserved, early-acting gene in vertebrate ovarian development. Endocrinology 144, 3237-3243 (2003).

13. Wang, D. S. et al. Foxl 2 up-regulates aromatase gene transcription in a female-specific manner by binding to the promoter as well as interacting with ad4 binding protein/steroidogenic factor 1. Mol. Endocrinol. 21, 712-725 (2007).

14. Pisarska, M. D., Barlow, G. \& Kuo, F. T. Minireview: roles of the forkhead transcription factor FOXL2 in granulosa cell biology and pathology. Endocrinology 152, 1199-1208 (2011)

15. Ayers, K. L. et al. RNA sequencing reveals sexually dimorphic gene expression before gonadal differentiation in chicken and allows comprehensive annotation of the W-chromosome. Genome Biol. 14; R26 (2013).

16. Ayers, K. L. et al. Identification of candidate gonadal sex differentiation genes in the chicken embryo using RNA-seq. BMC Genom. 16, 704 (2015).

17. Salter, D. et al. Gene insertion into the chicken germ line by retroviruses. Poult. Sci. 65, 1445-1458 (1986).

18. Bosselman, R. A. et al. Germline transmission of exogenous genes in the chicken. Science 243, 533-535 (1989).

19. Salter, D. \& Crittenden, L. Artificial insertion of a dominant gene for resistance to avian leukosis virus into the germ line of the chicken. Theor. Appl. Genet. 77, 457-461 (1989).

20. Harvey, A. J. \& Ivarie, R. Validating the hen as a bioreactor for the production of exogenous proteins in egg white. Poult. Sci. 82, 927-930 (2003).

21. Lu, Y., Lin, C. \& Wang, X. PiggyBac transgenic strategies in the developing chicken spinal cord. Nucleic Acids Res. 37, e141 (2009).

22. Park, T. S. \& Han, J. Y. PiggyBac transposition into primordial germ cells is an efficient tool for transgenesis in chickens. Proc. Natl. Acad. Sci. U. S. A. 109, 9337-9341 (2012).

23. Nakata, T., Ishiguro, M., Aduma, N., Izumi, H. \& Kuroiwa, A. Chicken hemogen homolog is involved in the chicken-specific sexdetermining mechanism. Proc. Natl. Acad. Sci. U. S. A. 110, 3417-3422 (2013).

24. Tanaka, R., Izumi, H. \& Kuroiwa, A. Androgens and androgen receptor signaling contribute to ovarian development in the chicken embryo. Mol. Cell Endocrinol. 443, 114-120 (2017).

25. Aduma, N., Izumi, H., Mizushima, S. \& Kuroiwa, A. Knockdown of DDX4 decreases the number of germ cells in male and female chicken embryonic gonads. Reprod. Fertil. Dev. 31, 847-854 (2018).

26. Park, T. S., Lee, H. J., Kim, K. H., Kim, J. S. \& Han, J. Y. Targeted gene knockout in chickens mediated by TALENs. Proc. Natl. Acad. Sci. U. S. A. 111, 12716-12721 (2014).

27. Oishi, I., Yoshii, K., Miyahara, D., Kagami, H. \& Tagami, T. Targeted mutagenesis in chicken using CRISPR/Cas9 system. Sci. Rep. 6, 23980 (2016).

28. Taylor, L. et al. Efficient TALEN-mediated gene targeting of chicken primordial germ cells. Development 144, 928-934 (2017). 
29. van de Lavoir, M. C. et al. Germline transmission of genetically modified primordial germ cells. Nature 441, 766-769 (2006).

30. Naito, M., Harumi, T. \& Kuwana, T. Long-term culture of chicken primordial germ cells isolated from embryonic blood and production of germline chimaeric chickens. Anim. Reprod. Sci. 153, 50-61 (2015).

31. EI-Samee, L. D. A., EI-Wardany, I., Ali, N. G. \& Abo-EI-Azab, O. M. A. Egg quality, fertility and hatchability of laying quails fed diets supplemented with organic zinc, chromium yeast or mannan oligosaccharides. Intl. J. Poult. Sci. 11, 221-224 (2012).

32. OECD. Test No. 223: Avian acute oral toxicity test, OECD guidelines for the testing of chemicals, Section 2, OECD Publishing. 2010.

33. Olszańska, B., Stepińska, U. \& Perry, M. M. Development of embryos from in vitro ovulated and fertilized oocytes of the quail (Coturnix coturnix japonica). J. Exp. Zoo.l 292, 580-586 (2002).

34. Mizushima, S. et al. The birth of quail chicks after intracytoplasmic sperm injection. Development 141, 3799-3806 (2014).

35. Ono, T. et al. A complete culture system for avian trasngenesis, supporting quail embryos from the single-cell stage to hatching. Dev. Biol. 161, 126-130 (1994).

36. Itoh, Y. et al. Dosage compensation is less effective in birds than in mammals. J. Biol. 6, 2 (2007).

37. Arnold, A. P., Itoh, Y. \& Melamed, E. A bird's-eye view of sex chromosome dosage compensation. Annu. Rev. Genomics Hum. Genet. 9, 109-127 (2008).

38. Zhang, S. O., Mathur, S., Hattem, G., Tassy, O. \& Pourquie, O. Sex-dimorphic gene expression and ineffective dosage compensation of Z-linked genes in gastrulating chicken embryos. BMC Genomics 11, 13 (2010).

39. Sekido, R. \& Lovell-Badge, R. Sex determination involves synergistic action of SRY and SF1 on a specific Sox9 enhancer. Nature 453, 930-934 (2008).

40. De Santa Barbara, P. et al. Direct interaction of SRY-related protein SOX9 and steroidogenic factor 1 regulates transcription of the human anti-Müllerian hormone gene. Mol. Cell Biol. 18, 6653-6665 (1998).

41. Giuili, G., Shen, W. \& Ingraham, H. The nuclear receptor SF-1 mediates sexually dimorphic expression of Mullerian Inhibiting Substance, in vivo. Development 124, 1799-1807 (1997).

42. Arango, N. A., Lovell-Badge, R. \& Behringer, R. R. Targeted mutagenesis of the endogenous mouse mis gene promoter. Cell 99, 409-419 (1999).

43. Nachtigal, M. W. et al. Wilms' tumor 1 and Dax-1 modulate the orphan nuclear receptor SF-1 in sex-specific gene expression. Cell 93, 445-454 (1998).

44. Johnson, P. A., Kent, T. R., Urick, M. E. \& Giles, J. R. Expression and regulation of anti-mullerian hormone in an oviparous species, the hen. Biol. Reprod. 78, 13-19 (2008).

45. Monniaux, D. et al. Regulation of anti-Müllerian hormone production in domestic animals. Reprod. Fertil. Dev. 25, 1-16 (2012).

46. Viger, R., Mertineit, C., Trasler, J. \& Nemer, M. Transcription factor GATA-4 is expressed in a sexually dimorphic pattern during mouse gonadal development and is a potent activator of the Mullerian inhibiting substance promoter. Development 125, 2665-2675 (1998).

47. Shen, W. H., Moore, C. C., Ikeda, Y., Parker, K. L. \& Ingraham, H. A. Nuclear receptor steroidogenic factor 1 regulates the müllerian inhibiting substance gene: a link to the sex determination cascade. Cell 77, 651-661 (1994).

48. Smith, C. A., Smith, M. J. \& Sinclair, A. H. Gene expression during gonadogenesis in the chicken embryo. Gene 234, 395-402 (1999).

49. Yang, L. V., Nicholson, R. H., Kaplan, J., Galy, A. \& Li, L. Hemogen is a novel nuclear factor specifically expressed in mouse hematopoietic development and its human homologue EDAG maps to chromosome 9q22, a region containing breakpoints of hematological neoplasms. Mech. Dev. 104, 105-111 (2001).

50. Hirst, C. E. et al. Sex reversal and comparative data undermine the $\mathrm{W}$ chromosome and support Z-linked DMRT1 as the regulator of gonadal sex differentiation in birds. Endocrinology 158, 2970-2987 (2017).

51. Fridolfsson, A. K. \& Ellegren, H. A simple and universal method for molecular sexing of non-ratite birds. J. Avian Biol. 30, 116-121 (1999).

52. Ainworth, S. J., Stanley, R. L. \& Evans, D. J. R. Developmental stages of the Japanese quail. J. Anat. 216, 3-15 (2010).

53. Li, B. \& Dewey, C. N. RSEM: accurate transcript quantification from RNA-Seq data with or without a reference genome. $B M C$ Bioinformatics 12, 323 (2011).

54. Langmead, B. \& Salzberg, S. L. Fast gapped-read alignment with Bowtie 2. Nat. Methods 9, 357 (2012).

55. Sun, J., Nishiyama, T., Shimizu, K. \& Kadota, K. TCC: an R package for comparing tag count data with robust normalization strategies. BMC Bioinformatics 14, 219 (2013).

56. Feng, J. et al. GFOLD: a generalized fold change for ranking differentially expressed genes from RNA-seq data. Bioinformatics 28, 2782-2788 (2012).

57. Mandric, I. et al. Fast bootstrapping-based estimation of confidence intervals of expression levels and differential expression from RNA-Seq data. Bioinformatics 33, 3302-3304 (2017).

58. Jones, P. et al. InterProScan 5: Genome-scale protein function classification. Bioinformatics 30, 1236-1240 (2014).

59. Klopfenstein, D. V. et al. GOATOOLS: a python library for gene ontology analyses. Sci. Rep. 8, 10872 (2018).

\section{Acknowledgments}

This work was supported by JSPS KAKENHI Grant Number 16H06279 (PAGS) and 18K19317.

\section{Author contributions}

M.O., T.I. and A.K. designed the study; M.O. and T.I. performed informatic analyses; S.M. and S.M. performed sample collection and expression analyses; M.S. and Y.S. performed RNA-seq analyses; M.O., S.M., M.S. and A.K. wrote the manuscript; M.O., S.M., T.I., S.M. and A.K. discussed the results. All authors reviewed the manuscript.

\section{Competing interests}

The authors declare no competing interests.

\section{Additional information}

Supplementary information is available for this paper at https://doi.org/10.1038/s41598-020-77094-y.

Correspondence and requests for materials should be addressed to A.K.

Reprints and permissions information is available at www.nature.com/reprints.

Publisher's note Springer Nature remains neutral with regard to jurisdictional claims in published maps and institutional affiliations. 
(c) (i) Open Access This article is licensed under a Creative Commons Attribution 4.0 International cc) License, which permits use, sharing, adaptation, distribution and reproduction in any medium or format, as long as you give appropriate credit to the original author(s) and the source, provide a link to the Creative Commons licence, and indicate if changes were made. The images or other third party material in this article are included in the article's Creative Commons licence, unless indicated otherwise in a credit line to the material. If material is not included in the article's Creative Commons licence and your intended use is not permitted by statutory regulation or exceeds the permitted use, you will need to obtain permission directly from the copyright holder. To view a copy of this licence, visit http://creativecommons.org/licenses/by/4.0/.

(C) The Author(s) 2020 